\title{
The Magnetic Fields of the Universe and Their Origin
}

\author{
S. A. Colgate \& H. Li
}

Los Alamos National Lab, T-6, MS B288, Los Alamos, NM 87545, U.S.A.

\begin{abstract}
Recent rotation-measure observations of a dozen or so galaxy clusters have revealed a surprisingly large number of magnetic fields whose estimated energy and flux are, on average, $\sim 10^{58}$ ergs and $\sim 10^{41} \mathrm{G} \mathrm{cm}^{2}$, respectively. These quantities are so much larger than any coherent sums of individual galaxies within the cluster that an efficient galactic dynamo is required. We associate these fields with single AGNs within the cluster and, therefore, with all galaxies during their AGN phase. Only the central, massive black hole (BH) has the necessary binding energy, $\sim 10^{61}$ ergs. Only the accretion disk during the $\mathrm{BH}$ formation has the winding number, $\sim 10^{11}$ turns, necessary to make the gain and magnetic flux. We present a model of a $\mathrm{BH}$ accretion-disk dynamo that might create these magnetic fields, where the helicity of the $\alpha-\Omega$ dynamo is driven by star-disk collisions. The back reaction of the saturated dynamo forms a force-free field helix that carries the energy and flux of the dynamo and redistributes them within the clusters.
\end{abstract}

\section{Introduction}

The problem of understanding the origin of large-scale, galactic magnetic fields has been with us for over forty years. There have been many papers and reviews on the galactic and extragalactic magnetic fields (see Moffatt 1978; Parker 1979; Krause \& Radler 1980; Ruzmaikin, Sokoloff, \& Shukurov 1989; Wielebinski \& Krause 1993; Beck et al. 1996; Zweibel \& Heiles 1997; Kulsrud 1999), and observational reviews (see Miley 1980; Bridle \& Perley 1984; Krönberg 1994), including the observations themselves (e.g., Perley, Bridle, \& Willis 1984; Taylor et al. 1990; Taylor \& Perley 1993; Eilek et al. 1984).

Recent rapid progress in observational work on galaxy clusters has revealed a surprising result. The intracluster medium (ICM) appears to be definitely magnetized and, in many cases, perhaps is highly magnetized as convincingly argued by Eilek et al. (in preparation). Figure 1 presents one such example in the Hydra A Cluster as shown by the rotation-measure $\left(R_{m}\right)$ map made by Taylor \& Perley (1993). We will show in this article that the implied magnetic energy and flux estimated from extensive $R_{m}$ maps of a dozen or so galaxy clusters are so exceedingly large that conventional galactic-dynamo models may prove to be inadequate. We argue that a new source of energy and a different form of the galactic dynamo are required. 
As the rotation-measure observations of galaxy clusters are relatively new and some of them are (yet) unpublished by the observation teams, we will first explain some of the observation results in detail, then discuss their physical implications at length. In the second half of the article, we will propose a new paradigm related to AGN accretion disks and describe some of our recent efforts in understanding a sequence of physical processes revolving around the origin of cluster magnetic fields.

\section{Galactic and Extragalactic Magnetic Fields}

Faraday rotation measures, $R_{m}$, are shown to be consistent with six other physical interpretations of magnetic fields in our and nearby galaxies (star light polarization, interstellar Zeeman splitting, synchrotron emission, synchrotron polarization, and inferences by $\mathrm{X}$-ray emission and cosmic ray isotropy and pressure) (see Krönberg 1994 for a review), thus establishing $R_{m}$ as a reliable measure of galactic and extragalactic magnetic fields. Because of the existence of many self-illuminating as well as background sources, usually AGN, and the increasing sensitivity of radio detection, $R_{m}$ has become the recognized measure of extragalactic magnetic fields (Krönberg 1994; Taylor, Barton, \& Ge 1994; Ge \& Owen 1994; Krause \& Beck 1998).

\subsection{Magnetic Flux and Energy in Galaxy Clusters}

Recently, high-quality $R_{m}$ maps of self-illuminating sources of galaxy clusters where the distances are known have become available (for example, Taylor \& Perley 1993; Eilek et al., in preparation). An important quantity that has received less discussion in these papers is the magnitude of the magnetic flux and energy.

Figure 1 shows the $R_{m}$ map of the region illuminated by Hydra A in the cluster (courtesy of Taylor \& Perley 1993). The largest, single region of highest field in this map has approximately the following properties: the size $L \simeq 50 \mathrm{kpc}$ and $B \simeq 33 \mu \mathrm{G}$, derived on the basis that the field is patchy and is tangled on a $4 \mathrm{kpc}$ scale. This leads to startling estimates of flux, $F \approx B L^{2} \simeq 8 \times 10^{4} \mu \mathrm{G} \mathrm{kpc}^{2}$, and energy, $W=\left(B^{2} / 8 \pi\right) L^{3} \simeq 4 \times 10^{59} \mathrm{ergs}$, assuming that the tangled field is only confined to the $50 \mathrm{kpc}$ region. If this is extended to the whole cluster which is $\sim 500 \mathrm{kpc}$, then the implied flux and energy are correspondingly larger by a factor of 100 and $10^{3}$, respectively. A similar conclusion can be reached when a larger sample of $R_{m}$ of galaxy clusters are analyzed using the data presented in Eilek et al. (in preparation). In Table 1, we have reproduced part of the table given in Eilek et al. and added two columns where the approximate flux and energy are calculated assuming that the fields are partially tangled or in loops.

Furthermore, the estimated values of fluxes and energies are most likely to be the minimum of the actual magnetic fields existing in the galaxy clusters. Faraday rotation depends upon the component of the field strength along the line of sight, $B_{\|}$, the distance along the line of sight, $Z_{o}$, and the electron density, $n_{e}$. Estimates of $n_{e}$ can be made from the X-ray emission measurements of the clusters with a typical accuracy of $\sim 20 \%$, and it varies by factors of 2 to 4 over the region of the source but otherwise is nearly uniform, and clumping is small (Taylor et al. 1994). If the field is folded in any fashion so that regions of 
Table 1. A list of cluster core parameters and their estimated magnetic fluxes and energies. Mean magnetic field is taken to be $\sim$ $\sqrt{3} \times\left\langle B_{\|}\right\rangle$. Data in the first three columns are taken from Eilek et al. (in preparation).

\begin{tabular}{lcccc}
\hline Source & $\begin{array}{c}\text { Size } \\
(\mathrm{kpc})\end{array}$ & $\begin{array}{c}\left\langle B_{\|}\right\rangle \\
(\mu \mathrm{G})\end{array}$ & $\begin{array}{c}\left\langle B^{2} L^{3} / 8 \pi\right\rangle \\
\left(10^{58} \mathrm{ergs}\right)\end{array}$ & $\begin{array}{c}\left\langle B L^{2}\right\rangle \\
\left(10^{41} \mathrm{G} \mathrm{cm}^{2}\right)\end{array}$ \\
\hline A 400 & 100 & 2.9 & 3 & 5 \\
A 1795 & 7 & 18 & 0.03 & 0.17 \\
A 2052 & 8 & 17 & 0.05 & 0.17 \\
A 2029 & 10 & 1 & 0.0003 & 0.02 \\
A 2199 & 30 & 15 & 2 & 0.3 \\
A 2634 & 140 & 1.9 & 30 & 7 \\
A 4059 & 10 & 69 & 0.15 & 1 \\
Cyg A & 70 & 15 & 25 & 1.5 \\
Hydra A & 50 & 33 & 40 & 15 \\
Virgo A & 3 & 35 & 0.01 & 0.05 \\
\hline \hline
\end{tabular}

oppositely directed field are in the line of sight, then the observed $R_{m}$ will be smaller than that if the same field lines were straightened out into one direction. In other words, $R_{m}$ is a minimum measure of $B_{\|}$.

To put the above numbers in perspective, for a typical galaxy like ours, e.g., with $1 \mathrm{kpc}$ thickness, $3 \mathrm{kpc}$ Homberg radius, and a field of $\sim 3 \mu \mathrm{G}$, the magnetic flux and energy are roughly $10^{38} \mathrm{G} \mathrm{cm}^{2}$ and $4 \times 10^{52}$ ergs, respectively. One observes that the flux and energy given in Table 1 range from close to the Hydra A limit to no more than $10^{2}$ times that of a typical galaxy.

The magnitude of the implied fluxes and energies are so large, $\times 10^{3}$ and $\times 10^{6}$ respectively, compared to these quantities within standard galaxies that their origin requires a new source of energy and a different form of the dynamo than previous galactic models. These minimum energies are sometimes even larger than the baryonic binding energy of galaxies $\left(\sim 2 \times 10^{58} \mathrm{ergs}\right)$. The extremely large fluxes also seem out of reach via amplification by ordinary galaxy rotations in a Hubble time.

Next, we discuss the difficulty with using turbulence to create these nearly uniform, highly correlated and coherent regions of $R_{m}$ as seen in Figure 1. We then discuss the still greater difficulty of creating the total magnetic energy of the cluster based upon a turbulence-dynamo model.

\subsection{Turbulent Versus Coherent Fields}

It has been suggested by a number of people (Eilek 1999; DeYoung 1980; Ruzmaikin, Sokolov, \& Shukurov 1989; Goldman \& Rephaeli 1991; Jaffe 1980) that the entire cluster is uniformly turbulent due to Rayleigh-Taylor instabilities during matter in-fall into the cluster, and that this turbulence drives the cluster dynamo creating the fields. The problems with this interpretation are the total magnetic energy, the magnitude of the turbulence, the strength of the fields, the 
apparent correlation of $R_{m}$ maps with single AGN structures, and, finally, the limited number of rotations of the cluster in a Hubble age.

Because of the small rotation rate of the typical cluster, $\sim 100 \mathrm{~km} \mathrm{~s}^{-1}$, the available rotation energy is small, $\sim 10^{-2}$ of the cluster binding energy, which has a thermal velocity of $\sim 10^{3} \mathrm{~km} \mathrm{~s}^{-1}$. So, applying the turbulent model to Hydra $\mathrm{A}$ implies a magnetic energy $10^{3}$ greater than the rotational energy. Therefore, the dynamo must be of the $\alpha^{2}$ type where fields are generated on the small scale, yet, as Taylor et al. (1994) point out, the fields of Hydra A and A1795 reverse on the different sides of the core, requiring coherence on scales of $100 \mathrm{kpc}$. Since this reversal is correlated with the structure of the source, and since the energy generated at the small scale is small compared to the turbulence input and the turbulent input should be small compared to the binding energy (DeYoung 1992), we believe that all these factors point to random, localized sources of magnetic energy of size $>10^{60} \mathrm{ergs}$. This is probably too demanding for turbulence.

Furthermore, it will be difficult to produce the large-scale, coherent $R_{m}$ regions which have been observed in Hydra A (Figure 1, northern region) and several other galaxy clusters (Eilek et al., in preparation). This is because, in a turbulent plasma, the emission, the $R_{m}$, and the degree of polarization should all be statistically symmetric. Despite the unlikelihood of all these factors conspiring to create both a pattern and a nearly uniform $R_{m}$, one observes in many $R_{m}$ maps of AGN, mostly in clusters, a distinct match in the $R_{m}$ pattern with the jet-like pattern of emission. Particularly, the sign of the average $R_{m}$ in several cases reverses across a symmetry plane through the core of the AGN (Taylor \& Perley 1993). The size of the regions of uniform $R_{m}$ correlates strikingly with the size of the jet as a function of distance from the nucleus. We interpret this correlation as due to the source of the field being the AGN jet as opposed to a turbulent $\alpha-\Omega$ dynamo in the cluster as a whole.

\subsection{Average Field Structure}

Using serendipitous, polarized background sources (and, therefore, random lines of sight through random clusters) Clark, Krönberg, \& Böhringer (1999) have made $\sim 80 R_{m}$ measurements. Their observations have produced a boundary of the typical cluster in $R_{m}$ such that the average field is $\sim 3 \mu \mathrm{G}$ out to a radius of $R_{\text {cluster }} \simeq 300 \mathrm{kpc}$. The magnetic flux and energy, $10^{4} \mu \mathrm{G} \mathrm{kpc}^{2}$ and $10^{60} \mathrm{ergs}$, are then similar to the largest structure already discussed in Hydra A. If each galaxy of a typical cluster with $\simeq 50$ large galaxies contributes a high-field region during its AGN phase, then the probability of intersecting such a region of area that is $\simeq 1 \%$ of the cluster is roughly $\simeq 5 \times 10^{-3}$, so that, in 100 lines of sight, the probability of intersecting a Hydra-like region of an AGN in a cluster is $\simeq 50 \%$. This is not inconsistent with the variability they observed. Finally, we note that the large degree of polarization observed in these sources, $\sim 50 \%$, indicates that the rotation source and emission source cannot be in the same location (Burn 1966; Taylor 1991), otherwise polarized emission from various depths in the source would undergo different degrees of rotation and hence emerge depolarized. Therefore, in any model, the Faraday screen and the emission source must be related and even congruent in order that the screen and, hence, $R_{m}$ be correlated with the core of the AGN. 


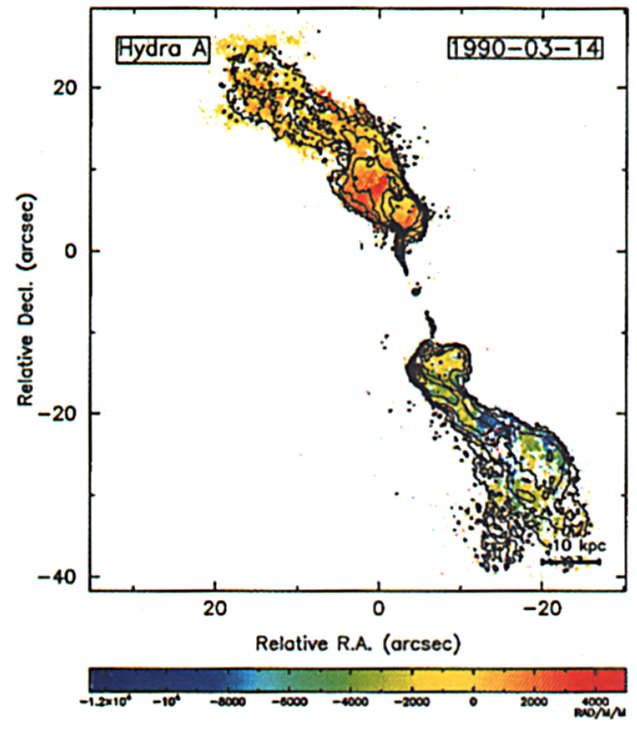

Fig.1. Faraday rotation, color, and intensity contours of an AGN, Hydra Cluster, $B=33$ micro $G, W=10^{\wedge} 60$ ergs, curtesy

(Taylor \& Perley '93).

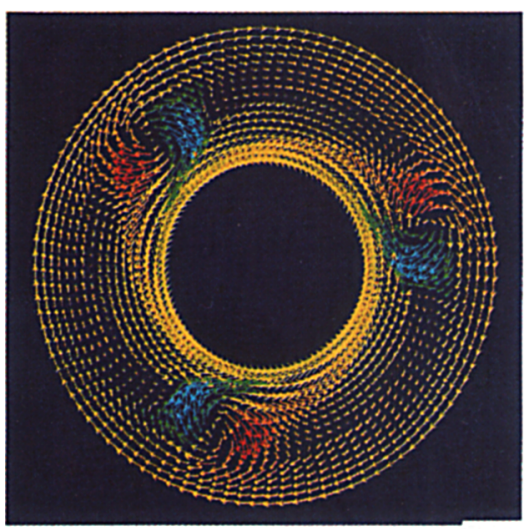

Fig. 2. The excitation of Rossby waves in linear theory in Keplerian flow is caused by a radial pressure gradient. The non-linear growth produces co-rotating Rossby vortices. Radial velocity pertubations are color coded against a Keplerian background flow in yellow.
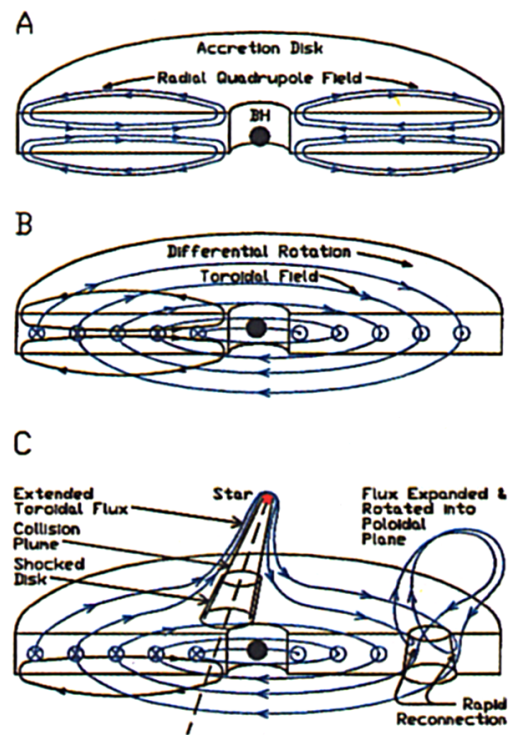

Fig. 3 The alpha -omega dynamo in the $\mathrm{BH}$ accretion disk. A: poloidal quadrupole field is wrapped up into a stronger toroidal field, B. A star-disk collision, C, carries a loop of toroidal flux above the plane. It is rotated into the poloidal plane by angular momentum. The poloidal flux is added to the initial quadrupole.

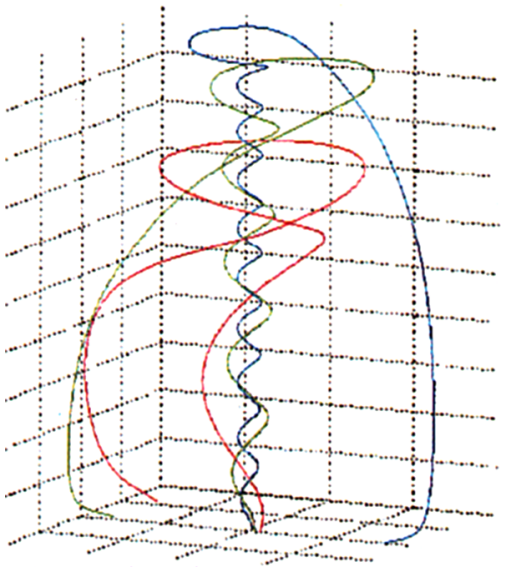

Fig. 4. A force free minimum energy helix is formed by the differential winding of the magnetic fluxfoot prints of the quadrupole field attached to the accretion disk. 


\subsection{Black Hole Accretion Disk as the Engine}

Purely based on the energetics, the accretion disk around a supermassive black hole in an AGN offers an attractive site for the production of magnetic fields. The accessible binding energy of the black hole is $\sim 10^{8} M_{\odot} c^{2} \sim 10^{61} \mathrm{ergs}$, and the winding number of the disk forming the $\mathrm{BH}$ of nearly every galaxy is $N_{w} \sim 5 \times 10^{10}$ at $10 R_{g}$, where $R_{g}$ is the $\mathrm{BH}$ horizon ( $\left.\sim 1 \mathrm{AU}\right)$. Using the canonical numbers thought to apply to AGN disks, the BH dynamo flux can be $F_{\mathrm{BHdyn}} \simeq B_{\mathrm{BHdyn}} \pi R_{\mathrm{BHdyn}}^{2} N_{w} \simeq 10^{43} \mathrm{G} \mathrm{cm}^{2}$, where we have used $B_{\mathrm{BHdyn}} \simeq$ $10^{4} \mathrm{G}$ at $L_{A G N} \sim 10^{46} \mathrm{ergs} \mathrm{s}^{-1}$ and $R_{\mathrm{BHdyn}} \simeq 10 R_{g} \approx 10^{14} \mathrm{~cm}$. Both the flux and energy from this simple analysis are $\sim 10$ times the maximum observed values. No other source of energy is likely to be sufficient by many orders of magnitude. Therefore, it is much more reasonable to assume that every AGN, both within and external to clusters, produces the magnetic energy and flux that we observe in this extreme case from the binding energy released in the accretion disk forming the central $\mathrm{BH}$. This implies that every galaxy contains a $\mathrm{BH}$ where $\sim 90-95 \%$ of the accessible binding energy is transformed into magnetic energy during its AGN phase by an accretion-disk dynamo. On the average, this flux and energy are distributed throughout the universe as force-free fields, and only a small fraction (5-10\%) of the magnetic energy is dissipated in the form of the AGN spectra, thus explaining the problem of the missing AGN luminosity (Richstone 1998; Krolik 1999). In this picture, a larger fraction of the magnetic energy is dissipated where the brightest AGNs are seen in galaxy clusters, because only in the clusters is a sufficient gas density retained by the gravity of the cluster such that this density confines the field, increasing the fraction of the magnetic energy that is dissipated. For most galaxies external to dense clusters, a small fraction of this magnetic energy is dissipated as the AGN radiation, a small fraction remains in the galaxy, and the bulk of the magnetic energy and flux is distributed in the walls and voids of the universe.

\section{Astrophysical Requirements and Progress with a Model}

The sequence of phenomena that can explain this astonishing, extragalactic magnetic flux and energy must start with an accretion disk forming a massive, central, galactic BH. This in turn presumes an answer to an equally enigmatic question, namely, the formation of these massive, galactic BHs themselves (Begelman et al. 1989; Rees 1999). By focusing on the transport of angular momentum, we believe that the flat rotation-curve mass distribution can be explained as a plausible result of any nonlinear collapse of an initial, gaseous, baryonic density fluctuation by hierarchical tidal torquing (Newman \& Wasserman 1999). The $\mathrm{BH}$ forms from this mass distribution when the Rossby-vortex torque mechanism supersedes tidal torquing and an accretion disk forms. All this mass then collapses to a $\mathrm{BH}$. The flat rotation curve, $M \propto R$, results in $\Sigma \propto R^{-1}$. When this thickness reaches $\Sigma \simeq 100$ to $1000 \mathrm{~g} \mathrm{~cm}^{-2}$, heat is confined for several revolutions, and the Rossby-vortex instability initiates at $M_{\text {disk }} \sim 10^{7}$ to $10^{8} M_{\odot}$. Finally, the dynamo-produced fields then supersede the previous torque mechanisms. 


\subsection{The Rossby-vortex Torque Mechanism}

We have predicted and demonstrated analytically and numerically how a new instability in Keplerian flow, the Rossby-vortex instability, can grow (Lovelace et al. 1999; $\mathrm{Li}$ et al. 2000; $\mathrm{Li}$ et al., in preparation[a]). The production of vortices is shown in Figure 2. This instability produces torque and thus transports angular momentum within an accretion disk by purely hydrodynamical means via the interaction of large, two-dimensional, corotating Rossby vortices. The enhanced transport of angular momentum by corotating vortices is recognized in rotational atmospheric flows (Staley \& Gall 1979) and in laboratory measurements of the Ranque-Hilsch tube (Hilsch 1947; Fröhlingsdorf \& Unger 1999; Colgate \& Buchler 1999).

\subsection{The Dynamo, Star-Disk Collisions, and Helicity}

A coherent dynamo can form in a Keplerian accretion disk because of the large, azimuthal velocity shear, provided that there exists a robust source of nonaxisymmetric helicity. Classically, turbulence has been invoked to explain this helicity using the mean-field dynamo theory, but we know of no way to create this degree of turbulence, with vertical motions, hydrodynamically in an accretion disk, because hydrodynamic turbulence alone is damped in an accretion disk (Balbus \& Hawley 1998). The magnetic instability of Balbus \& Hawley will lead to turbulence, but the magnitude of the turbulence is orders of magnitude too small compared to the Keplerian stress. Instead, we have identified a new, robust source of helicity driven by star-disk collisions by a small mass fraction $\left(\sim 10^{-3}-10^{-4}\right)$ of pre-galaxy-formation stars. The Keplerian shear and a stardisk collision with the twist-producing helicity is shown in Figure 3. We have demonstrated by laboratory flow-visualization experiments how plumes, driven in a rotating frame, counterrotate relative to the frame (Beckley \& Colgate 1998; Beckley et al., in preparation) and thus produce a robust and coherent helicity where flux is always added in the same direction and where the driving force is large compared to the Keplerian stress in the disk.

We have simulated the positive, exponential gain of both the quadrupole and dipole poloidal fields of such a dynamo with a vector potential code in 3-D, cylindrical coordinates, where the velocity field simulates both the Keplerian rotation and star-collision-produced plumes. We have observed a growth rate of $\sim 10 \%$ per revolution, two plumes per two revolutions, $R_{\text {plume }}=1 / 3 R_{\text {disk }}$, and with a magnetic Reynolds number, $R_{e y, \Omega, B}=100$ (Pariev, Colgate, \& Finn, in preparation).

\subsection{The Saturation of the Dynamo and the Formation of the Helix}

With positive gain and large winding number, the dynamo will saturate regardless of how small the seed field is. Since the helicity does not depend on turbulence, it will not be subject to turbulent $\alpha$-quenching at the small scale (Vainshtein \& Cattaneo 1992; Vainshtein \& Rosner 1991). Furthermore, since the stars maintain virial velocity, their velocity is supersonic relative to the disk, and the resulting shock stress is large. At the back-reaction limit, the field grows until the torque of the field affects the Keplerian motion, and the accessible $\mathrm{BH}$ binding energy is converted into magnetic energy. The progressive loss of this flux is a force-free, helical, Poynting magnetic flux which 
we identify as collimated AGN jets. We have investigated the field topology of these twisted, helical flux surfaces by integrating the Grad-Shafranov equations for a force-free axisymmetric field with a Keplerian distribution of winding number ( $\mathrm{Li}$ et al., in preparation[b]) as shown in Figure 4 . Since the field decreases as $B_{\text {helix }} \propto 1 / R$, the pressure at large radius as the helix extends to Mpcs becomes of the order of the IGM (intergalactic medium), and the outer boundary of the helix is self-collimating (Lynden-Bell 1996). The energy carried by this helix at a mean radius near the $\mathrm{BH}, R_{d y n} \simeq 10 R_{B H}$, is the accessible energy of accretion or $\dot{M}_{B H} c^{2} / 10=\left(B_{\text {helix }}^{2} / 8 \pi\right)\left(100 \pi R_{B H}^{2}\right)$ or $B_{\text {helix }} \simeq 10^{4} \mathrm{G}, I=5 R_{\text {helix }} B_{\text {helix }}=5 \times 10^{18} \mathrm{amps}, V_{\text {potential }}=10^{20}$ volts, and $I \times V_{\text {potential }}=10^{39}$ watts $=10^{46} \mathrm{erg} \mathrm{s}^{-1}$. General relativity inside the innermost stable orbit will add additional energy (Blandford \& Znajek 1977; Livio, Ogilvie, \& Pringle 1999).

\section{4. $J_{\|}$Reconnection and Acceleration}

The distribution of this flux in the universe occurs by partial tearing-mode reconnection producing the minimum-energy Taylor state (Taylor 1986). The total flux is conserved, but a fraction of the energy is dissipated in the tearingmode, $J_{\|}$reconnection. The resulting $E_{\|}$acceleration of the current carriers produces the emission that we associate with AGN.

Acknowledgments. We are indebted to Richard Lovelace, Howard Beckley, Vladimir Pariev, John Finn, Mike Warren, Dave Westpfahl, Van Romero, Ragnar Ferrel, and Warner Miller for direct contributions to this project and to very many more who have contributed in discussions, criticisms, and encouragements. HL acknowledges the support of an Oppenheimer Fellowship. This research is supported by the DOE, under contract W-7405-ENG-36.

\section{References}

Balbus, S. A., \& Hawley, J. F. 1998, Rev. Mod. Phys., 70, 1

Beck, R. et al. 1996, ARA\&A, 34, 155

Beckley, H. F. \& Colgate, S. A. 1998, APS, DFD, Abst. 5253

Beckley, H. F. et al., in preparation

Begelman, M. C., et al. 1989, in NATO ASI Series C, 290, Theory of Accretion Disks, eds. F. Meyer, W. Duschl, J. Frank, \& E. Meyer-Hofmeister, (Dordrecht: Kluwer), 373

Blandford, R. D., \& Znajek, R. L. 1977, MNRAS, 179, 433

Bridle, A. H., \& Perley, R. A. 1984, ARA\&A, 22, 319

Burn, B. F. 1966, MNRAS, 133, 67

Clark, T., Krönberg, P. P., \& Böhringer, H. 1999, preprint

Colgate, S. A., \& Buchler, R. J. 1999, in Ann. NY Acad. Sci., 14th Florida Workshop in Astrophysical Turbulence and Convection, eds. R. Buchler $\&$ H. Kantrup (New York: ANYAS), in press

DeYoung, D. S. 1980, ApJ, 241, 81

DeYoung, D. S. 1992, ApJ, 386, 464 
Eilek, J. A. 1999, in MPE Report 271, Diffuse Thermal and Relativistic Plasma in Galaxy Clusters, eds. H. Bohringer, L. Feretti, \& P. Schuecker (Garching: MPE), 71

Eilek, J. A., Burns, J. O., Odea, C. P., \& Owen, F. N. 1984, ApJ, 278, 37

Eilek, J. A. et al., in preparation

Fröhlingsdorf, W., \& Unger, H. 1999, Int. J. of Heat and Mass Transfer, 42, 415

Ge, J-P., \& Owen, F. N. 1994, AJ, 108, 1523

Goldman, I., \& Rephaeli, Y. 1991, ApJ, 380, 344

Hilsch, R. 1947, Rev. Sci. Instr., 18, 108

Jaffe, W. 1980, ApJ, 241, 925

Krause, F., \& Beck, R. 1998, A\&A, 335, 789

Krause, F., \& Radler, K-H. 1980, Mean Field Electrodynamics and Dynamo Theory (Berlin: Akademie-Verlag)

Krolik, J. H. 1998, Active Galactic Nuclei (Princeton: Princeton Univ. Press)

Krönberg, P. P. 1994, Prog. Phys., 57, 325

Kulsrud, R. M. 1999, ARA\&A, 37, 37

$\mathrm{Li}, \mathrm{H}$, , et al. 2000, ApJ, in press

$\mathrm{Li}, \mathrm{H}$., et al., in preparation[a]

$\mathrm{Li}, \mathrm{H}$., et al., in preparation[b]

Livio, M., Ogilvie, G. I., \& Pringle, J. E. 1999, ApJ, 512, 100

Lovelace, R. V. E., Li, H., Colgate, S. A., \& Nelson, A. F. 1999, ApJ, 513, 805

Lynden-Bell, D. 1996, MNRAS, 279, 389

Miley, G. K. 1980, ARA\&A, 18, 165

Moffatt, H. K. 1978, Magnetic Field Generation in Conducting Fluids (Cambridge: Cambridge Univ. Press)

Newman, W. I., \& Wasserman, I. 1999, ApJ, 354, 411

Pariev, V., Colgate, S. A., \& Finn, J. M., in preparation

Parker, E. N. 1979, Cosmical Magnetic Fields: Their Origin and Their Activity (Oxford: Claredon Press)

Perley, R. A., Bridle, A. H., \& Willis, A. G. 1984, ApJS, 54, 291

Rees, M. J. 1999, astro-ph/9912346, preprint

Richstone, D. 1998, Nature, 395, 14

Ruzmaikin, A. A., Sokolov, D., \& Shukurov, A. 1989, MNRAS, 241, 1

Staley, D. O., \& Gall, R. L. 1979, J. Atmos. Sci., 36, No. 6, 973

Taylor, G. B. 1991, Ph.D. thesis, UCLA

Taylor, G. B., Barton, E. J., \& Ge, J. 1994, AJ, 107, 1942

Taylor, G. B., \& Perley, R. A. 1993, ApJ, 416, 554

Taylor, G. B., Perley, R. A., Inoue, M., Kato, T., Tabara, H., \& Aizu, K. 1990, ApJ, 360, 41

Taylor, J. B. 1986, Rev. of Mod. Phys., 58, 741

Vainshtein, L. I., \& Cattaneo, F. 1992, ApJ, 393, 165

Vainshtein, S. I., \& Rosner, R. 1991, ApJ, 376, 199 
Wielebinski, R., \& Krause, F. 1993, A\&A Rev., 4, 449

Zweibel, E. G., \& Heiles, C. 1997, Nature, 385, 131 